\title{
CARACTERIZACIÓN MATERIAL Y PROCESO DE CONSERVACIÓN DE LA COLECCIÓN DE DOCUMENTOS ÁRABES MANUSCRITOS DEL ARCHIVO HISTÓRICO PROVINCIAL DE GRANADA
}

\author{
CHARACTERIZATION OF MATERIALS AND CONSERVATION \\ PROCESS OF THE COLLECTION OF ARABIC MANUSCRIPT \\ DOCUMENTS OF GRANADA ARCHIVO \\ HISTÓRICO-PROVINCIAL
}

\author{
T. EsPejo Arias \\ I. Lazarova Stoytcheva \\ (Universidad de Granada) \\ D. CAmpillo García \\ (Universidad de Murcia)
}

A. Durán Benito

M.C. JiMÉNEZ DE HARO

Instituto de Ciencia de Materiales de Sevilla

(CSIC-Universidad de Sevilla)

\begin{abstract}
El texto que presentamos recoge las principales conclusiones que han derivado de los estudios sobre la Colección de documentos árabes del Archivo Histórico Provincial de Granada. Compuesta principalmente por documentos de carácter jurídico, el análisis del contenido de cada uno de ellos en relación con la materialidad del soporte y las tintas así como la coincidencia en el diseño y planificación de la página revelan el uso de idénticos protocolos de ejecución. Esta investigación nos está permitiendo, además, establecer coincidencias relevantes relacionadas con los procesos de elaboración del papel entre los últimos años de permanencia de los árabes en la Península Ibérica y los primeros tras la incorporación del territorio a la Corona de Castilla y, sobre todo, con el modo de ejecutar el documento de archivo en sus formas externa e interna.
\end{abstract}

Palabras clave: manuscritos; actas notariales; al-Andalus; papel.
This paper presents the main conclusions of our study of the Arabic documents preserved in the Archivo Histórico Provincial of Granada. The analysis of the contents of each document, the materials and inks used in their support, and also the similarities of the page layout reveals the use of identical production protocols. Furthermore, this research also enables us to establish important similarities between the paper production processes in the latter stages of the Islamic rule in the Iberian Peninsula and the earlier stages of its incorporation in the Crown of Castilla - in particular in relation to how the archival documents were issued, both externally and internally.

Key works: Manuscripts; Notarial deeds; alAndalus; Paper. 


\section{Introducción ${ }^{1}$}

Inéditos hasta el momento, en el Archivo Histórico Provincial de Granada se custodian una serie de manuscritos árabes de gran importancia tanto por su carácter y temática como por su data. La colección pertenece al fondo de la Administración Periférica del Estado y, dentro de éste, a la Comisión Provincial de Monumentos HistóricoArtísticos de Granada.

Según los datos que constan en el registro del Archivo ${ }^{2}$, los documentos objeto de este estudio fueron hallados en Lanjarón (Granada) en 1838 y donados por D. José de Castro y Orozco (1808-1869), marqués de Gerona y miembro ilustre de la Comisión de Monumentos Histórico-Artísticos de Granada, al Museo Arqueológico de esta ciudad en 1866 entrando a formar parte del patrimonio de esta Comisión - caracterizada por su continua actuación para la recogida y salvaguarda de libros - conformando una biblioteca de gran interés. En 1993 la biblioteca, junto con el resto del fondo documental de la institución, ingresó en el Archivo Histórico Provincial de Granada ${ }^{3}$ convirtiéndose en uno de sus fondos bibliográficos más destacables.

De acuerdo con la información aportada por Amalia Zomeño, la colección está formada por quince documentos de carácter notarial datados en Granada entre 1475 y 1498 y seis manuscritos sin data que parecen formar parte de un archivo privado. Los primeros contienen veintiuna escrituras judiciales que se corresponden con diez

${ }^{1}$ Este trabajo se ha desarrollado dentro del marco de investigación del proyecto «Caracterización de los materiales de manuscritos árabes de la Península Ibérica para la elaboración de un corpus documental» (P08-HUM 04188, Proyectos de Investigación de Excelencia, convocatoria 2008) y de acuerdo con los protocolos de descripción, análisis de materiales y determinación de los procesos de ejecución desarrollados en el proyecto «Aplicación de tecnologías de análisis específicas para el conocimiento de materiales y la mejora de los procesos de conservación de los manuscritos árabes de la Península Ibérica (ss. X-XVII)» (MAT2008-02008MAT, Plan nacional I+D+I 2008-2011). Agradecimientos al contrato post-doctoral JAE Doc 088 CSIC concedido a uno de los autores.

2 AHPGR, Comisión Provincial de Monumentos, Inventario del Museo Arqueológico 1870 , leg. 1841 , núm. 57.

3 Aunque el Archivo Histórico Provincial de Granada fue creado a nivel estatal por Orden Ministerial de 20 de mayo de 1994, ya desde 1991 empezó a funcionar como una unidad independiente del Archivo de la Real Chancillería de Granada que había asumido sus funciones desde 1969, siendo gestionado por la Consejería de Cultura de la Junta de Andalucía. Para más información: Martín López, R.E., "Archivo Histórico Provincial de Granada", en R. Marín y P. Enríquez (eds.), Guía de archivos históricos de Granada, Granada, 2001. 
contratos de compraventa, dos pliegos particionales, un mandato judicial y un legado. El resto se sitúa dentro de un contexto privado ${ }^{4}$ : una carta y una serie de notas informales sobre diferentes asuntos, como precios, cantidades adeudadas, etc., así como un pequeño tratado sobre cómo hacer las oraciones rituales. A excepción de los documentos privados, el estudio de la colección revela una serie de particularidades vinculadas por su forma y producción que se repiten en todos los casos y que están directamente relacionados con el contenido del documento y el mensaje explícito que sustentan ${ }^{5}$. La coincidencia en el empleo de materiales y la observación de ciertos elementos que conciernen al diseño y la planificación del documento en su forma externa, junto con el uso de protocolos de redacción precisos, demuestran el empleo de formularios establecidos para cada uno de los diferentes actos jurídicos expresados ${ }^{6}$. Así, estos documentos públicos se caracterizan fundamentalmente por la utilización de una sola hoja de papel para cada uno de los actos jurídicos que se desarrollan, la concepción de la página a partir de un amplio margen derecho que desplaza la caja de texto hacia la izquierda, la coincidencia en el espacio de interlineado o el empleo de una única tinta negra que concuerda en el texto y en la firma del primer alfaquí y difiere - cromáticamente o en su composición - con la firma del segundo (Figura 1).

Para el estudio de la colección se ha seguido un protocolo de trabajo basado en la cumplimentación individualizada para cada documento de una ficha de caracterización ${ }^{7}$ que recoge un área de descripción catalográfica y una serie de apartados relacionados con el examen organoléptico del documento, su composición material o

${ }^{4}$ El contenido de los manuscritos está siendo estudiado por Amalia Zomeño, investigadora del Instituto de Lenguas y Culturas del Mediterráneo y Oriente Próximo (CSIC) y Antonio Peláez, investigador de la Escuela de Estudios Arabes de Granada (CSIC), quienes amablemente nos han facilitado esta información. Nuestro más sincero agradecimiento por su colaboración.

5 Torres Ibáñez, D., “¿Qué es un manuscrito andalusí?”, en T. Espejo y J.P. Arias (eds.), El Manuscrito Andalusí. Hacia una denominación de origen, Junta de Andalucía. Conserjería de Cultura, Granada, 2008, pp. 79-80.

${ }^{6}$ Este dato coincide con los estudios llevados a cabo por A. Zomeño en otras colecciones granadinas. Cf. Zomeño, A., "Del escritorio al tribunal. Estudio de los documentos notariales de la Granada nazarí", en J.P. Monferrer y M. Aldón, Grapheion Códices, manuscritos e imágenes. Estudios filológicos e históricos, Córdoba, 2003, pp. 75-102.

7 Espejo, T. y Beny, A., "Protocolo de descripción del manuscrito andalusí", en Espejo y Arias, El Manuscrito Andalusí, pp. 87-134. 

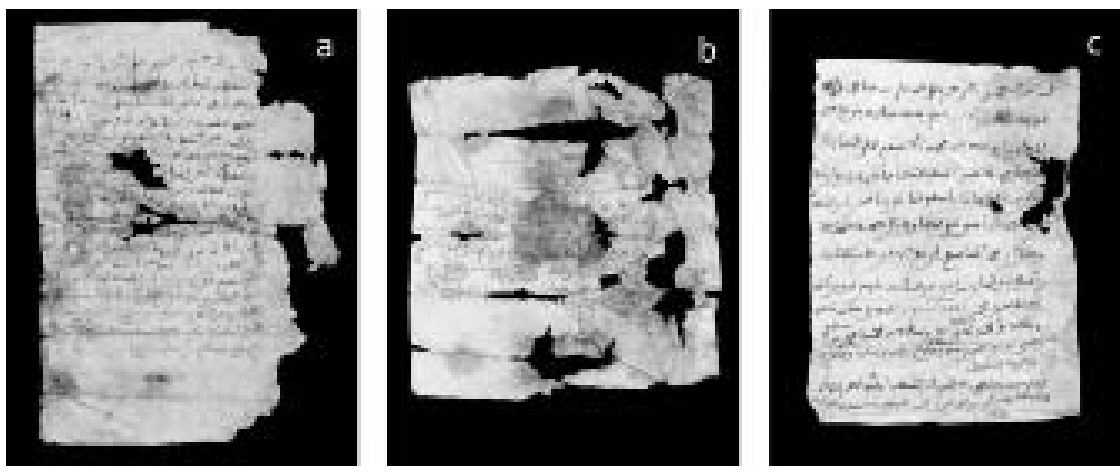

Figura 1. (a) Ms.7,

(b) Ms. 11, (c) Ms. 19. Antes de la restauración

la impaginación entre otros datos. La referencia asignada en este trabajo para cada documento coincide con la signatura dada por el Archivo para cada uno.

\section{Examen organoléptico}

En todos los documentos estudiados el papel utilizado como soporte para la escritura ha sido manufacturado de acuerdo con el modelo de fabricación occidental. No obstante, aunque en ellos se ha empleado la forma metálica y presentan marca de agua, sus características lo alejan de la calidad del denominado papel italiano: son papeles más gruesos, con desigual distribución de la pasta y, frecuentemente, con defectos de formación de la hoja. También se han observado corondeles separados no siempre de manera regular y, ocasionalmente, alabeados o reparados, así como puntizones deformados y arqueados. Sobre el origen o la procedencia de este papel no tenemos datos objetivos aunque sí hemos encontrado características similares en otros estudios que realizamos en la actualidad ${ }^{8}$.

A excepción de los manuscritos 18 y 21 que, dentro del contexto privado, se han concebido en modo singulion, el resto de los documentos que conforman la colección han sido redactados sobre una

${ }^{8}$ Se ha observado la utilización de papel de similares características en un porcentaje muy elevado de documentos pertenecientes a la Colección de manuscritos árabes del Fondo Antiguo de la Biblioteca del Hospital Real de la Universidad de Granada. 
hoja de papel independiente. El estudio de la verjura, de la filigrana y el examen de los bordes - barbados o no- demuestran que ninguno de los documentos coincide en formato con las de un pliego de papel completo. Sus dimensiones oscilan entre $149 \times 100 \mathrm{~mm}$ (manuscrito 15) y $325 \times 215 \mathrm{~mm}$ (manuscrito 5) y el espesor de las hojas entre 0,10 y $0,30 \mathrm{~mm}$ siendo, en la mayoría de los documentos, bastante irregular. Las diferencias más pronunciadas las encontramos en el manuscrito 14 donde las medidas fluctúan entre 0,16 y $0,24 \mathrm{~mm}$.

La observación con luz transmitida permite comprobar que la pasta papelera está bien trabajada, no presenta impurezas, restos de trapo o hebras desiguales. La disposición de la pulpa es bastante homogénea aunque se ha detectado mayor concentración en la zona de las cadenetas (manuscrito 2) y en puntos localizados de la hoja. En algunas zonas, la acumulación de pulpa coincide con una diferencia de pigmentación y un aumento en la higroscopicidad del papel que está directamente relacionado con un ataque biológico por acción de microorganismos, por lo que no podemos asegurar si se trata efectivamente de una mayor concentración de la pasta o de una desnaturalización del soporte. De la misma forma, son evidentes defectos en la formación de la hoja tales como burbujas de aire o marcas de arrastre de pulpa debidas al movimiento de la pasta durante el proceso de manufactura. Sin embargo, en términos generales, podemos afirmar que el desfibrado ha sido eficiente y el grado de refino bueno.

La verjura del papel es evidente en todos los casos y nos lleva a determinar el empleo de la forma occidental cuya característica principal es la obtención de un pliego de verjura regular con corondeles y puntizones ordenados. A pesar de que este principio se cumple en todos los documentos, encontramos alguna alteración en la disposición y el número de corondeles para cada documento, llegando en algún caso - manuscrito 9- a no ser perceptibles o a estar ligeramente arqueados o muy deformados, con anomalías en forma de doble corondel, tal y como se observa en el manuscrito 2. El número de puntizones contabilizados en $20 \mathrm{~mm}$ también difiere según el documento variando de 17 en los manuscritos 5 y 11 , a 26 en el manuscrito 3.

De los veintiún documentos examinados hemos constatado la presencia de filigrana en seis - manuscritos $3,10,15,17,18$ y $21-$, siendo la mano o guante la principal protagonista. El estado de con- 
servación del soporte o el formato del documento imposibilitan la observación total de la marca de agua que aparece fragmentada en todos los casos. La única excepción la encontramos en el manuscrito 21 en el que la mano se sitúa en el interior de un doble círculo junto a una estrella $\mathrm{y}$, ambas, bajo una grafía en la que puede leerse $M A I O R$. Aunque sin data expresa, este dato puede indicarnos una cronología posterior. En este caso, su situación en el centro del bifolio - ligeramente desplazada a la izquierda sobre el folio 2-y la disposición horizontal de los corondeles nos indica que el pliego original debió doblarse in quarto dando lugar a este formato.

El formato de los documentos, la situación de la filigrana en el corte del folio y la disposición horizontal de los corondeles en los manuscritos 3, 15, 17 y 20 indican que, igual que en el caso anterior, estas hojas pudieron haber sido plegadas y cortadas in quarto. Sin embargo, esto no ocurre en todos los casos, mientras que en el manuscrito 10 su situación en el centro del folio y la disposición vertical de los corondeles nos lleva a pensar que se trata de un pliego de papel cortado in folio. Por último, el manuscrito 18 (singulion) ha sido cortado in quarto, pero plegado in octavo (Figura 2).

\section{Impaginación}

En todos los documentos que conforman la colección el texto se ha elaborado en una sola columna ${ }^{9}$ y se ha constatado el empleo de la punta seca para la confección de una marca que señala el inicio de las líneas de texto - en el manuscrito 21 aparece también delimitando el final de la línea- así como el empleo de la tinta negra, ya sea para la configuración del cuerpo del texto, las firmas o las notas al margen. Sin embargo, existen claras diferencias en relación con la impaginación si analizamos cada uno de los tipos apuntados. En los singuliones el texto se presenta más o menos centrado en el folio mientras que en los documentos concebidos en una sola hoja salta a

${ }^{9}$ Los manuscritos 5 y 10 parecen contener notas o ser algún tipo de listado. Una primera aproximación a la observación de la página nos ha llevado a pensar en la disposición del texto en dos columnas. Sin embargo, el estado de conservación de los documentos impide determinar con exactitud el contenido de los mismos y por consiguiente, la ordenación, por lo que estos datos están siendo revisados y serán confirmados en un estado más avanzado de nuestra investigación. 

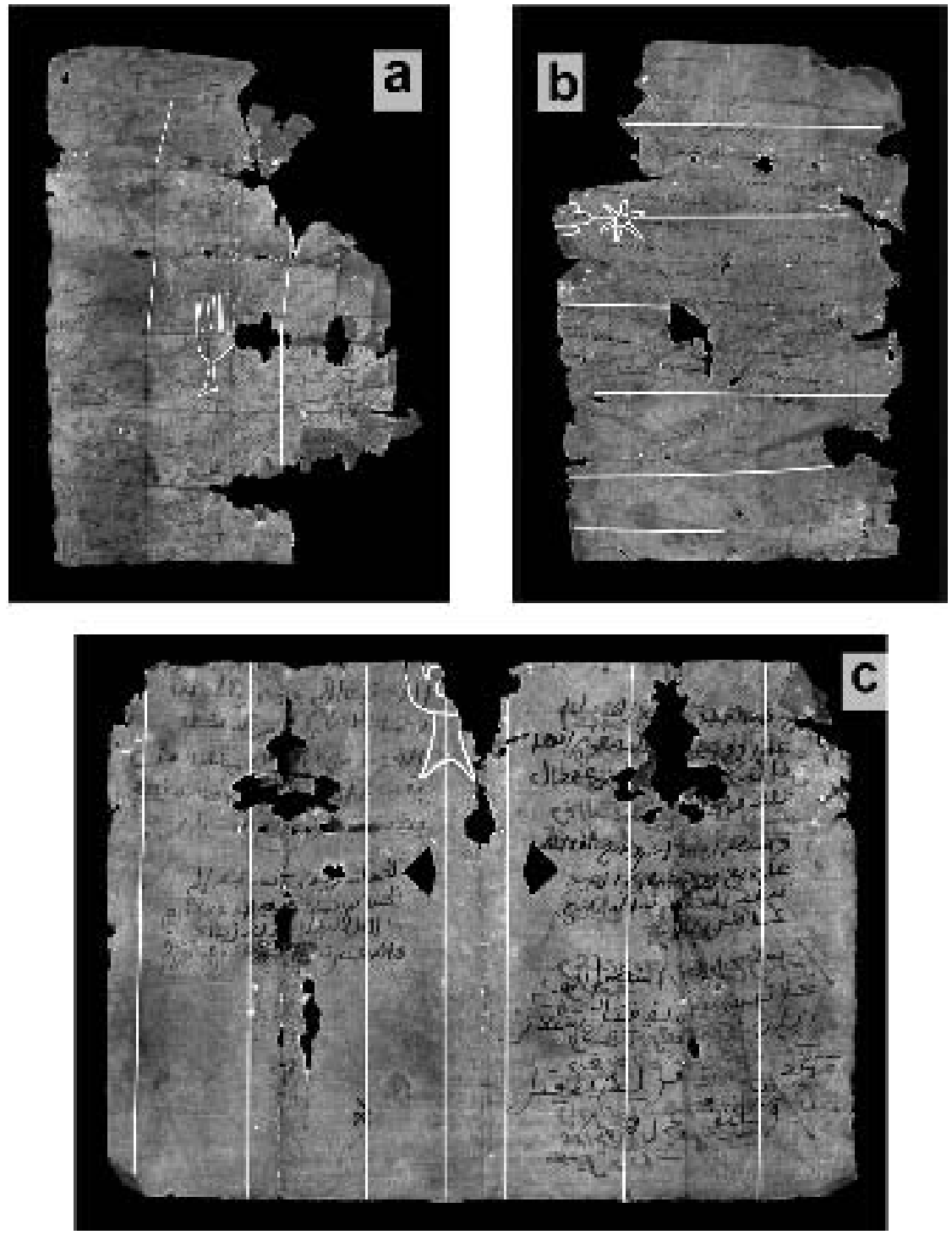

Figura 2. Diferentes ejemplos del corte o doblado del papel en función de la disposición de la filigrana: (a) corte in folio (Ms.10), (b) corte in quarto (Ms. 3), (c) plegado in octavo (Ms.18). 
la vista la diferencia entre el margen derecho, de dimensiones considerables, y el izquierdo en el que el texto termina a sangre con el borde del papel; el número de líneas y el interlineado también varía considerablemente en cada uno de ellos.

De acuerdo con las marcas existentes en la actualidad, todos los documentos presentan una serie de pliegues transversales que difieren en número según el documento, desde un mínimo de tres en el manuscrito 9 hasta un máximo de nueve en el manuscrito 17. No tenemos información sobre la intencionalidad de este plegado, si está en relación con el carácter del documento, con la finalidad para la que fue concebido o responde a alguna medida de conservación, almacenamiento o para su transporte; en cualquier caso, dado que aparece localizado en la mayor parte de los manuscritos, nos parece oportuna su toma en consideración.

Apoyando el carácter y tipología de estos documentos cabe llamar la atención sobre los orificios intencionados que se observan en el margen derecho de los dos folios que constituyen el manuscrito 18. Cotejando este documento con otros de origen cristiano procedentes del mismo archivo o del Archivo de la Real Chancillería de Granada, el ejemplo más cercano que hemos encontrado se refiere al sistema de hojas horadadas, un recurso corriente utilizado en documentación de tipo notarial y en registros cancillerescos desde la segunda mitad del siglo XV y, sobre todo, en el siglo XVI. Éste consiste en tomar el documento o unidad documental y efectuar, normalmente en el ángulo superior izquierdo - en manuscritos cristianos-, un corte en forma circular que permitiera pasar a través de él un hilo de cáñamo que, anudado en cabeza, servía de sujeción del conjunto. Este procedimiento permitía unir tanto un bloque completo de documentación como diferentes unidades, cuadernos o folios que luego se podrían ensamblar conjuntamente. Su objetivo era conservar con efectos jurídicos las copias generadas por la institución con la posibilidad de realización de un cartulario que las reuniera y conservara.

\section{Caracterización material}

La caracterización material se ha llevado a cabo de manera coordinada entre conservadores de la Universidad de Granada e investigadores del Instituto de Ciencia de Materiales (CSIC) de Sevilla, 
usando diferentes técnicas para el análisis y la identificación tanto de las fibras de papel como de los componentes de las tintas presentes en los manuscritos.

Las técnicas experimentales utilizadas en este caso han sido la microscopía óptica - empleando un microscopio Nikon HOPTIHOT-, la microscopía electrónica de barrido (SEM) utilizando un microscopio electrónico HITACHI S-4800 al que se ha acoplado un analizador de energía dispersiva de rayos-X (EDX) para el análisis elemental de las tintas, y la espectroscopía de infrarrojos. Esta última técnica ha sido muy poco empleada en muestras de papel o textiles a pesar de que resulta muy adecuada para distinguir las distintas clases de fibras en función de las bandas de absorción que pueden ser asignadas a los diferentes componentes específicos de las fibras como son la celulosa, hemicelulosa, pectina y lignina ${ }^{10}$. Para estos estudios se empleó un espectrómetro Nicolet 510 (Fuente: Globar, Detector: DTGS/KBr). Las muestras han sido molidas y preparadas en polvo con $\mathrm{KBr}(1 \mathrm{mg}$ de muestra en $50 \mathrm{mg}$ de $\mathrm{KBr}$ aproximadamente). Los espectros resultantes han sido recogidos en modo transmisión, en un rango de número de onda entre $4000-400 \mathrm{~cm}^{-1}$ y una resolución de $4 \mathrm{~cm}^{-1}$.

Los resultados derivados de los estudio de las fibras mediante microscopías óptica y electrónica ${ }^{11}$ revelan la presencia de lino en todos los casos (Figura 3). Se observa la existencia de algunas fibras dañadas y la presencia de actividad biológica (manuscritos 7 y 15).

Para distinguir las fibras mediante espectroscopía de infrarrojos se han calculado dos relaciones $\mathrm{R} 1=\mathrm{I}_{1595} / \mathrm{I}_{1105}$ y R2 $=\mathrm{I}_{1595} / \mathrm{I}_{2900}$. Las bandas situadas en el espectro a $1595 \mathrm{~cm}^{-1}$ hacen referencia a los grupos $\mathrm{C}=\mathrm{C}$ y se asignan a la lignina; las bandas a $1105 \mathrm{~cm}^{-1}$ al enlace glicosídico C-O-C de los polisacáridos de la celulosa; y las bandas a $2900 \mathrm{~cm}^{-1}$ vienen asociadas generalmente a materia orgáni-

${ }^{10}$ Espejo Arias, T., Durán Benito, A., López-Montes, A. y Blanc García, R., “Application of Analytical Technologies in the Study of Hispano-Arabic Paper Manuscripts. A Transition Model in $15^{\text {th }}$ Century Granada", en IPH Congress Book (International Association of Paper Historians. Hispano-Arabic Paper \& Paper Museums and their Significance), vol. 16, special edition 2006, pp. 17-27.

${ }_{11}$ Perry, D.R., Identification of Textile Materials, 7th edition, Manchester, 1985, p. 262; Lewin, M. y Pearce, E.M., Handbook of fiber chemistry, 2nd edition, New York, 1998, p. 1136.

Al-Qantara XXXII 2, julio-diciembre 2011, pp. 519-532 ISSN 0211-3589 


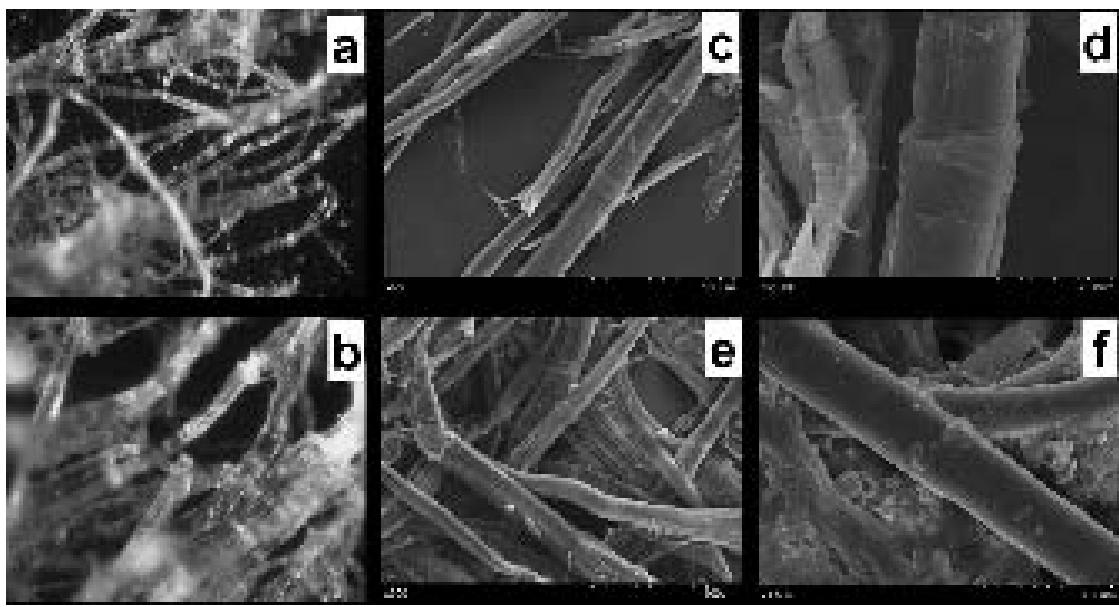

Figura 3. Micro-fotografías de fibras correspondientes a:

— Microscopía óptica (a) Ms. 3 (× 100), (b) Ms. $5(\times 200)$;

- Microscopía electrónica de barrido (c) Ms. 2, (d) Ms. 7, (e) Ms. 9, (f) Ms. 18

ca (grupos $-\mathrm{CH}_{3},-\mathrm{CH}_{2-}-\mathrm{CH}_{3}$, metilo y etilo) ${ }^{12}$. Dependiendo de los porcentajes teóricos de celulosa, hemicelulosa, pectina y lignina en las fibras y de la relación entre los valores R1 y R2, se han definido cuatro regiones que corresponden a las zonas de existencia de lino, yute, cáñamo y algodón (Figura 4). Los espectros obtenidos en el análisis de once de las muestras sirvieron para la medida cuantitativa de la intensidad de las bandas de absorbancia indicadas y el cálculo de las relaciones R1 y R2. Los resultados indican que todas las muestras corresponden a fibras de lino.

El análisis de las diferentes muestras de tinta negra mediante SEM-EDX evidencian la presencia de carbón (Figura 5, 5a) y hierro junto con azufre en un porcentaje elevado (Figura 5, 5b). Relacionar la presencia de carbono con la composición de la tinta en este tipo de muestras es difícil, dado que este elemento forma parte de la propia composición de las fibras que constituyen el papel. La presencia

${ }^{12}$ Garside, P. y Wyeth, P., "Identification of cellulosic fibres by FTIR spectroscopy", Studies in Conservation, 48 (2003), pp. 269-275; Espejo, T., Durán, A., López-Montes, A. y Blanc, R., "Microscopic and spectroscopic techniques for the study of paper supports and textile used in the binding of Hispano-Arabic manuscripts from Al-Andalus: A transition model in the 15th century", Journal of Cultural Heritage, 11 (2010), pp. 50-58. 


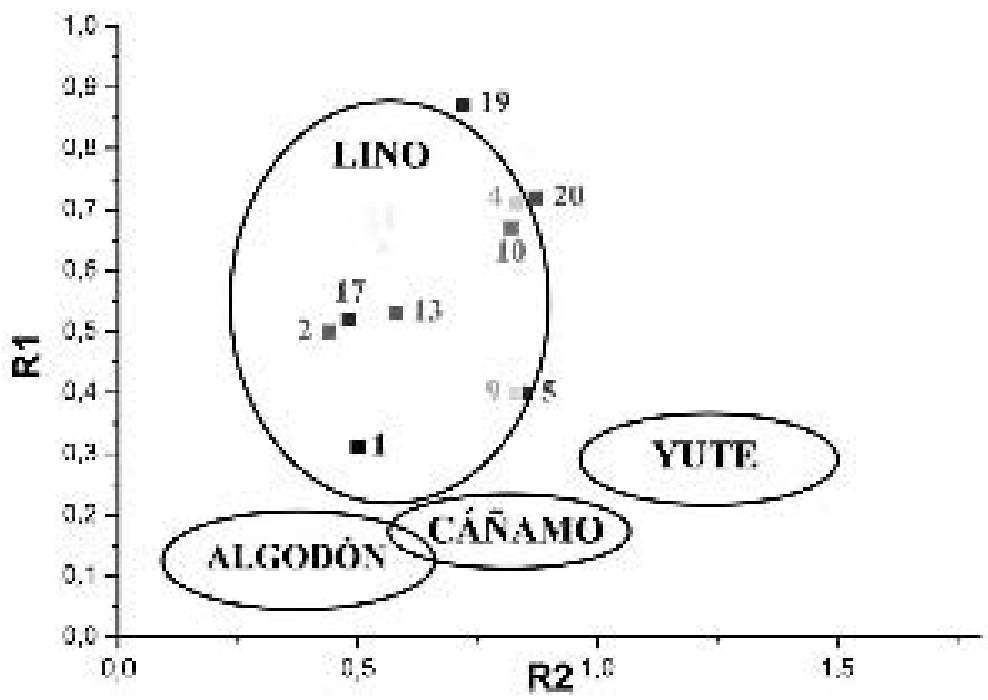

Figura 4. Representación R1-R2 de las once muestras estudiadas por espectroscopía de infrarrojos. Las zonas teóricas de existencia de cada tipo de fibra se indican en la figura

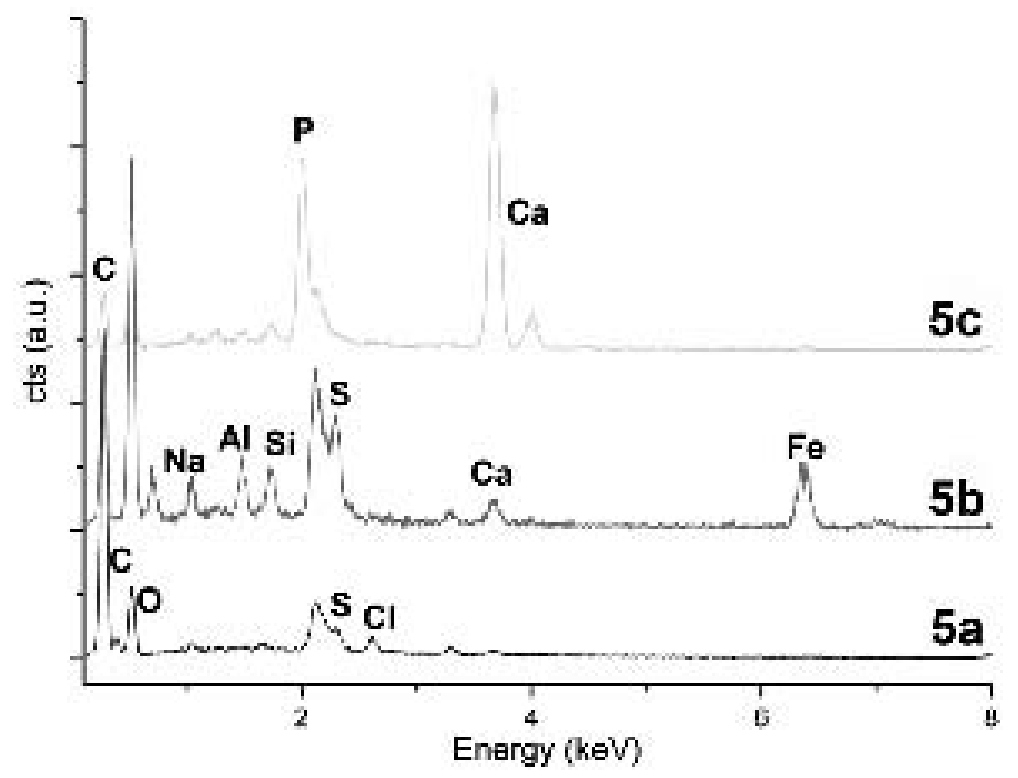

Figura 5. Espectros EDX correspondientes a las tintas negras:

(a) $\mathrm{t} 8 \mathrm{-a}, \quad$ (b) $\mathrm{t} 13-\mathrm{e}, \quad$ (c) t10-b.

Al-Qanțara XXXII 2, julio-diciembre 2011, pp. 519-532 ISSN 0211-3589 
de hierro y azufre demuestran claramente la utilización de una tinta metaloácida con base de hierro - tinta ferrogálica-. El fósforo $(\mathrm{P})$ (Figura 5, 5c) detectado en alguna de las muestras indica, además, el empleo de una tinta mixta elaborada añadiendo a la tinta ferrogálica un negro de huesos con el objeto de conseguir un negro más profundo. Por otra parte, en algunos análisis se detectó hierro junto con silicio y aluminio en gran proporción, lo que asociamos a la presencia de tierras con óxidos de hierro, capaces de proporcionar mayor cuerpo a la tinta. También han sido localizadas partículas de calcita, dolomita y yeso procedentes posiblemente de la manufactura propia del soporte.

\section{Estado de conservación y criterios de intervención para su conservación}

El grado de deterioro que presentaban los documentos previo a la intervención conservativa variaba considerablemente en función del manuscrito a tratar, pasando de un estado de conservación bastante aceptable - como era el caso del manuscrito 21 - hasta la pérdida de más del 90\% del documento, tal y como evidencian los fragmentos denominados como manuscrito 16. Entre ambos casos encontramos diversos estados intermedios; así, destacan los daños producidos por la humedad o por la acción biológica que han provocado manchas y variaciones cromáticas y han favorecido la desnaturalización de la celulosa, la solubilización de los aprestos y materias adhesivas y la pérdida de materia física. El empleo de tintas metaloácidas también es evidente en los manuscritos 2, 13, 14, 18 o 21 a través de testimonios que van desde la simple variación cromática producida por la proporción de los componentes y los procesos de ejecución, la presencia de halos característicos o la destrucción del soporte. La presencia de pliegues originales o de arrugas y deformaciones como consecuencia de una mala conservación así como importantes cúmulos de suciedad, completan el cuadro de alteraciones.

Una vez valorado el estado de conservación, la intervención para la recuperación y conservación de la colección se ha llevado a cabo según los criterios generales que rigen cualquier intervención en conservación y restauración de los bienes culturales. Siguiendo el mismo protocolo de trabajo adoptado para la investiga- 
ción y tratamiento de otras colecciones estudiadas, el principio adoptado como fundamental parte de la mínima intervención y el respeto por su integridad física y documental, documentando tanto gráfica como fotográficamente, los diferentes procesos llevados a cabo, respetando cualquier vestigio original y evitando aquellas intervenciones que pudieran alterar las características intrínsecas o formales de cada documento. Es por ello que los tratamientos aplicados han tenido como objetivo principal frenar el deterioro y devolver al documento las condiciones necesarias que le permitan recuperar su funcionalidad; se han evitado los tratamientos acuosos que pudieran implicar alteración de los componentes del papel y las tintas o variaciones en el acabado característico, sólo se ha llevado a cabo reintegración material del soporte en aquellos casos en los que resultaba imprescindible para el mantenimiento de la integridad física del documento y se han laminado sólo los documentos especialmente débiles. Por otra parte, se ha considerado necesario el establecimiento de adecuadas medidas de protección física para cada documento como ente independiente y parte de un conjunto que aseguren una correcta manipulación y almacenaje de la colección tras la intervención. De acuerdo con estas premisas y teniendo en cuenta que el grado de intervención para cada documento varía en función de su nivel de deterioro, los tratamientos aplicados han sido: limpieza; eliminación de arrugas, pliegues y deformaciones; reparación de desgarros; consolidación; laminación; reintegración de soporte; reproducción fotográfica digital y elaboración de sistemas de protección directa - en forma de sobres - para cada documento y de un contenedor rígido para el conjunto de la colección.

\section{Conclusiones}

En relación con el contenido y la impaginación, todos los documentos responden a una misma tipología. Su carácter jurídico y el mantenimiento de un mismo patrón de diseño pueden servir para plantear la posible existencia de una tipología de documentación notarial, en al-Andalus en este periodo, que emplea comúnmente el papel como soporte y que, además de emplear formularios establecidos, responde a protocolos concretos de ejecución. 
Las características que acabamos de exponer, las fuentes consultadas y el elevado número de ejemplares que se vienen localizando en otras colecciones nos llevan a pensar en la interrelación entre este modo de fabricación y la ejecución de los manuscritos por lo que no resultaría extraño pensar en la existencia de molinos que abastecieran de papel a las instituciones y que emplearan las técnicas y procedimientos de fabricación citados.

En cuanto a los aspectos materiales del documento, resaltamos el hecho de que el papel, a pesar de estar fabricado a la manera italiana, presenta elementos característicos del papel árabe tales como el espesor, el reparto poco homogéneo de la pulpa o el empleo exclusivo de la fibra de lino. Del mismo modo, las irregularidades observadas en relación con las marcas dejadas por los elementos de la forma utilizada en el proceso de fabricación lo alejan de la calidad atribuida a éstos y lo acercan a procedimientos más arcaicos de elaboración. La presencia de estos elementos confiere al material un aspecto peculiar que nos hace pensar en la posibilidad de que se trate de un papel de transición entre los procedimientos de elaboración a la manera árabe y los nuevos métodos cristianos. Unos procedimientos oscos indudablemente a tenor de los resultados, que serían fruto de las transferencias tecnológicas en los procesos de producción en una época de contrastadas influencias cruzadas. En cualquier caso, los datos obtenidos respecto a este último punto no son todavía determinantes en relación con el origen y la localización de la fabricación; son aspectos en fase de investigación sobre los que esperamos poder aportar información concluyente en futuras comunicaciones.

Finalmente, de los estudios llevados a cabo se ha concluido la determinación de los métodos y procedimientos más adecuados para la intervención en su conservación permitiendo recuperar una serie de documentos, desconocidos hasta el momento y ponerlos al servicio de los investigadores. Los resultados se pueden observar de primera mano en el Archivo Histórico Provincial de Granada.

Recibido: $28 / 01 / 2011$

Aceptado: 30/05/2011 